\title{
Pompe disease: clinical perspectives
}

This article was published in the following Dove Press journal:

Orphan Drugs: Research and Reviews

28 December 2016

Number of times this article has been viewed

\section{Juan Francisco Cabello'} Deborah Marsden²

'Genetics and Metabolic Disease Laboratory, Nutrition and Food Technology Institute (INTA), University of Chile, Santiago, Chile; ${ }^{2}$ Division of Genetics and Genomics, Boston Children's Hospital, Boston, MA, USA
Correspondence: Deborah Marsden Division of Genetics and Genomics, Boston Children's Hospital, 300 Longwood Ave, Boston, MA 0211 5, USA Tel +I 6173554695

Fax + I 6177300466 Email deborah.marsden@childrens. harvard.edu

\begin{abstract}
Pompe disease (acid alpha-glucosidase deficiency, OMIM 232300) is a rare lysosomal storage disorder due to autosomal recessive mutations in the GAA gene. It has also been called acid maltase deficiency and glycogen storage disease type II. There is a broad clinical presentation: the most severe form that presents in the first few months of life with cardiomyopathy and generalized muscle weakness that rapidly progresses to death from cardio-respiratory failure in the first year of life (infant-onset Pompe disease). A more slowly progressive disease, with little or no cardiac involvement, presents with proximal myopathy and/or pulmonary insufficiency, from the second year of life to late adulthood (late-onset Pompe disease). The recent development and introduction of enzyme replacement therapy with intravenous infusion of recombinant human acid alpha-glucosidase have made a major improvement in the morbidity and mortality of this disease. New therapies are also in development. With the availability of treatment, diagnostic methods have also improved, allowing for earlier recognition and potential early therapeutic intervention. The advent of newborn screening for Pompe disease may identify patients who can be treated before significant irreversible disease has occurred.
\end{abstract}

Keywords: Pompe disease, glycogen storage disease, lysosomal storage disease, enzyme replacement therapy, gene therapy, chaperone therapy, genotype/phenotype, newborn screening

\section{Introduction}

Pompe disease is an autosomal recessive inherited disorder of glycogen metabolism due to a deficiency of the lysosomal enzyme, acid alpha-glucosidase (GAA) (also called acid maltase), resulting from mutations in the GAA gene (EC 3.2.1.20). Historically, it has been classified as a glycogen storage disease (type II), an enzyme deficiency (acid maltase deficiency), and more recently as a lysosomal storage disease. A deficiency of the enzyme $^{1}$ results in neuromuscular symptoms, ranging from severe early-onset disease, with involvement primarily of the heart, proximal skeletal muscles, diaphragm, and intercostal muscles, leading to cardio-respiratory failure and death in infancy, to a later onset, more slowly progressive, limb-girdle muscular dystrophy with diaphragm involvement, leading eventually to wheelchair dependence, respiratory failure, and death in adulthood.

The disease was first described in 1932 by a Dutch pathologist, Johannes Pompe, when he noted ubiquitous deposition of glycogen in vacuoles in the heart and other tissues of a 7-month-old girl who had died from hypertrophic cardiomyopathy. In 1963, HG Cori classified it as glycogen storage disease type II and, following the discovery of the lysosome by de Duve, Hers determined that the intra-lysosomal glycogen deposition was secondary to a deficiency of acid alpha-1,4 glucosidase (acid maltase), thus submit your manuscript | www.dovepress.com

Dovepress

http://dx.doi.org//0.2147/ODRR.S69109
Orphan Drugs: Research and Reviews 2017:7 I-10

(c) (i) (5) 2017 Cabello and Marsden. This work is published and licensed by Dove Medical Press Limited. The full terms of this license are available at https://www.dovepress. cc) the work you hereby accept the Terms. Non-commercial uses of the work are permitted without any further permission from Dove Medical Press Limited, provided the work is properly attributed. For permission for commercial use of this work, please see paragraphs 4.2 and 5 of our Terms (https://www.dovepress.com/terms.php). 
the first known lysosomal storage disorder. Currently, there are over 40 disorders known to be due to a defect of one of the lysosomal hydrolase enzymes.

\section{Biochemistry/molecular genetics}

Acid alpha-1,4-glucosidase is a lysosomal enzyme that hydrolyzes the alpha-1,4 and alpha-1,6 linkages of its natural substrate, glycogen at acid $\mathrm{pH}$. The enzyme is primarily targeted to the lysosome via mannose-6-phosphate receptors. The enzyme appears to be expressed in most tissues, ${ }^{1}$ including skeletal muscle, diaphragm, heart, placenta, kidneys, and central nervous system (CNS), predominantly in the spinal cord and brain stem Schwann cells and neuronal cell, including the anterior horn cells.

To date, over 300 variations have been detected in the $G A A$ gene, ${ }^{2}$ which is mapped to $17 \mathrm{q} 25.2-25.3$, including 116 pathogenic mutations considered to be very severe, 91 potentially less severe, 23 less severe, and 18 potentially mild. The gene encodes an inactive precursor protein with a molecular mass of $\sim 110 \mathrm{kD}$ a that is transported to the prelysosomal and lysosomal compartments and further processed to 95,76 , and $70 \mathrm{kDa}$ fragments that can be seen on Western blots, and appear to be fully active and not produced by nonspecific proteolysis during the purification process. ${ }^{3}$ The severity of the mutations largely depends on where the mutations occur in the gene and the type of mutation. ${ }^{4}$ The combination of mutations in heterozygous patients may explain some of the phenotypic heterogeneity seen in this disease. Large deletions or nonsense mutations involving the coding area of the gene, or other critical areas for transcription, result in a truncated enzyme with essentially no activity. Patients who inherit two severe mutations have infant onset Pompe disease (IOPD). The combination of a severe mutation and a mutation that retains some transcriptional activity is usually associated with late-onset Pompe disease (LOPD). ${ }^{5,6}$ The most severe mutations result in no detectable protein on Western blot analysis, and therefore designated cross reactive immune material-negative (CRIMnegative). A normal length precursor protein that is not fully translated can also result in a nonfunctional enzyme.

A database is maintained for all reported mutations at Erasmus Medical Center in the Netherlands. ${ }^{7}$ The tables list pathogenic variants, with predicted severity. Nonpathogenic variants and variants of unknown significance are also listed. This information may be helpful in predicting genotypephenotype correlation in some cases. ${ }^{4}$

\section{Pathology}

In affected patients, the classic appearance on muscle biopsy is of a vacuolar myopathy with periodic-acid Schiff staining of areas that, on electron microscopy, are seen to be intralysosomal glycogen. Accumulated glycogen may also be seen in the cytoplasm and inter-fibrillary spaces. ${ }^{8}$ Massive accumulation eventually leads to rupture of the lysosome and large "glycogen lakes." This process has been thought to contribute to the muscle pathology by disrupting the contractile elements of the muscle cell leading to fibrosis and loss of function. ${ }^{9}$ More recently, it has been shown in the knockout mouse model, and also confirmed in humans, that autophagy (a normal process of degradation of lysosomal or other malfunctioning cellular components) in type II, fast twitch glycolytic muscle fibers leads to build up of autophagic granules and destruction of the fibers. Accumulation of autophagic material in these muscle fibers ${ }^{10,11}$ also appears to act as a sink for therapeutic recombinant enzyme, disrupting efficient delivery to the lysosomes. The type I slow twitch oxidative fibers appear to store less glycogen, but clear it more rapidly ${ }^{11}$ with enzyme replacement therapy (ERT).

In addition to autophagy, it has been recognized that mitochondrial dysfunction and abnormalities in calcium homeostasis ${ }^{12}$ may also contribute to the pathology. Intramitochondrial calcium has a buffering role, and overload may result in necrosis or apoptosis. Dysfunctional mitochondria can also contribute to cellular pathology due to increased production of reactive oxygen species and consequent damage.

Recently, Falk et $\mathrm{al}^{13}$ have reported that in the Pompe knockout mouse model there were both pre- and postsynaptic neuropathic changes in the neuromuscular junction tissues of skeletal muscle and diaphragm, suggesting that neuromuscular junction pathology may contribute to the decline in pulmonary and muscle function in Pompe disease. Small-fiber neuropathy may also contribute to the clinical phenotypes of both infant- and late-onset patients. ${ }^{14}$

\section{Epidemiology}

Pompe disease is an autosomal recessive disorder with an estimated worldwide incidence of $\sim 1 / 40,000,{ }^{1}$ although the precise number is not known as the disease can be difficult to diagnose and there may be larger numbers of unrecognized patients. Newborn screening programs (discussed later) may eventually be able to provide more accurate information. Data from the Pompe Registry ${ }^{15}$ indicate that $\sim 20 \%$ of patients have IOPD. There is a different incidence of Pompe disease in some populations, often with regional mutations, suggesting founder effects, especially in Taiwanese/Southern Chinese population, African-Americans, and the Druze population in Israel. 
It was thought for some time that the Taiwanese population had a higher incidence of Pompe disease, as the diagnosed patients were typically children. However, early newborn screening ${ }^{16}$ data suggest that the incidence of IOPD is $\sim 1 / 57,000$ and for LOPD 1/26,500, with an overall incidence of $\sim 1 / 40,000$, similar to other populations. Of the later-onset patients to date, most appear to be children. The majority of patients carry at least one copy of the severe p.Asp645Glu mutation, ${ }^{17}$ which is likely a founder mutation in this population. A pseudo-deficiency allele, p.[G576S; E689K], ${ }^{18,19}$ is relatively common in Asian populations and leads to falsely low measured enzyme activity. It was recently reported in $3.9 \%$ of Japanese. It has also been found in the Taiwanese population.

In African-Americans, ${ }^{1}$ the incidence is $\sim 1 / 14,000$, with a common severe mutation p.Arg854X, which is CRIMnegative.

In late-onset Caucasian patients, there is a high incidence of a leaky splice-site mutation, c.336-13T $>$ G, present in $~ 70 \%$ of alleles. ${ }^{6}$ It has been found in combination with many different mutations with a wide spectrum of clinical presentations.

\section{Clinical phenotypes}

Pompe disease can present anytime from the newborn period through late adulthood. Historically, it has been classified as infant-onset, presenting in the first year of life, juvenile-onset presenting from the second year of life through adolescence, and adult-onset later in life. In reality, however, it is a continuum of symptoms involving multiple systems. Patients who may be diagnosed later in life, because of neuromuscular and/or pulmonary symptoms, often recall symptoms in childhood that were not recognized as being pathologic. ${ }^{20}$ Other symptoms may be related to generalized involvement of smooth muscle, including vascular and intestinal systems (Table 1).

There is no definitive genotype-phenotype correlation; there have been rare reports of variable ages of presentation within the same family carrying the same mutations, ${ }^{21}$ such as one family with severe infant-onset symptoms in one

Table I Common features in late-onset Pompe disease

\begin{tabular}{l} 
Proximal muscle weakness \\
Gait instability, falling \\
Difficulty climbing stairs \\
Neck weakness \\
Scapular winging \\
Paraspinal muscle atrophy \\
Swallowing difficulty \\
Facial weakness, ptosis \\
\hline
\end{tabular}

generation and milder late-onset symptoms in another. The severity of symptoms and rate of progression of the disease may also depend on other epigenetic variables; ${ }^{22}$ however, in general, earlier onset of symptoms is associated with more severe and more rapidly progressive disease. ${ }^{20}$

Affected infants have been detected prenatally, especially in families with a previously affected infant, by finding cardiac enlargement on ultrasound. The average age of diagnosis is $\sim 4$ months. ${ }^{23}$ There is $<1 \%$ measureable enzyme activity, ${ }^{1}$ with $\sim 10 \%$ having no detectable enzyme activity (CRIM-negative), although the CRIM-negative rate may be higher in some consanguineous populations, such as the south Asians in the UK, ${ }^{40}$ where it can be almost $50 \%$.

Presenting symptoms are related to muscle weakness and/or cardiac involvement (Table 2). The infants appear to be cognitively normal. The majority of untreated infants (discussed below) die of cardio-respiratory failure by 1 year of age. A few atypical infants may survive into later infancy or childhood, with progressive muscle weakness, less severe heart involvement, but often requiring ventilator support.

The more common LOPD, also traditionally referred to as acid maltase deficiency, ${ }^{1}$ is essentially a form of limb-girdle muscular dystrophy. There is little or no cardiac involvement. These patients usually have residual enzyme activity of $10 \%-30 \%$. Symptoms can present any time after the first year of life and progress slowly. Surprisingly, $\sim 13 \%$ of late-onset patients have been shown to have $<1 \%$ residual enzyme activity, ${ }^{24}$ so there is not always a direct correlation between clinical phenotype and residual enzyme activity. The initial symptoms (Table 1) are usually related to proximal muscle weakness, ${ }^{20}$ with the lower limbs being more affected than the upper limbs. The weakness becomes progressively worse with later involvement of the intercostal muscles and the diaphragm, leading to respiratory failure and ventilator-dependence. Patients typically survive through the fourth of fifth decades. Occasionally, pulmonary symptoms can precede significant muscle weakness.

Table 3 provides the differential diagnosis.

Table 2 Common features in infant-onset Pompe disease

Hypotonia
Head lag
Respiratory distress
Macroglossia
Cardiomegaly
Cardiac failure
Feeding difficulty
Failure to thrive
Motor delay


Table 3 Differential diagnosis of Pompe Disease

\begin{tabular}{|c|c|}
\hline Infant-onset & non signs and symptoms \\
\hline $\begin{array}{l}\text { Spinal muscular atrophy } \\
\text { (Werdnig-Hoffmann) }\end{array}$ & $\begin{array}{l}\text { Hypotonia, progressive limb-girdle muscle } \\
\text { weakness, absent reflexes, feeding difficulties, } \\
\text { elevated creatine kinase (CK) }\end{array}$ \\
\hline $\begin{array}{l}\text { Congenital muscular } \\
\text { dystrophy }\end{array}$ & Severe hypotonia and muscle weakness \\
\hline Mitochondrial disorders & $\begin{array}{l}\text { Hepatomegaly, cardiomyopathy, myopathy, } \\
\text { elevated CK }\end{array}$ \\
\hline Peroxisomal disorders & Hypotonia, hepatomegaly \\
\hline Prader-Willi Syndrome & $\begin{array}{l}\text { Dysmorphic features, severe feeding } \\
\text { difficulties, absence of cardiomegaly }\end{array}$ \\
\hline $\begin{array}{l}\text { Idiopathic hypertrophic } \\
\text { cardiomyopathy }\end{array}$ & Biventricular hypertrophy \\
\hline Myocarditis & $\begin{array}{l}\text { Inflammation of the myocardium contributing } \\
\text { to cardiac enlargement }\end{array}$ \\
\hline Endocardial fibroelastosis & $\begin{array}{l}\text { Breathlessness, feeding difficulties, } \\
\text { cardiomegaly, heart failure }\end{array}$ \\
\hline Carnitine defici & Cardiomyopathy, muscle weakness \\
\hline \multicolumn{2}{|l|}{ Late-onset } \\
\hline $\begin{array}{l}\text { Proximal limb-girdle } \\
\text { muscular dystrophies }\end{array}$ & $\begin{array}{l}\text { Progressive muscle weakness in the pelvis, } \\
\text { legs, or shoulders, abnormal gait, elevated CK }\end{array}$ \\
\hline Danon disease & $\begin{array}{l}\text { Skeletal muscle myopathy, limb-girdle } \\
\text { muscle weakness, scapuloperoneal muscular } \\
\text { weakness, elevated CK }\end{array}$ \\
\hline $\begin{array}{l}\text { Becker muscular } \\
\text { dystrophy }\end{array}$ & $\begin{array}{l}\text { Progressive limb-girdle muscle weakness, } \\
\text { respiratory impairment, difficulty walking, } \\
\text { elevated CK }\end{array}$ \\
\hline Myastheni & Daily fluctuation, fatigue, ocular involvement \\
\hline Polymyositis & $\begin{array}{l}\text { Progressive, often symmetrical, muscle } \\
\text { weakness, difficulty swallowing, elevated CK }\end{array}$ \\
\hline
\end{tabular}

\section{Diagnosis}

Until recently, the diagnosis of Pompe disease has been made by measuring enzyme activity using a fluorometric assay with the artificial substrate, 4-methylumbelliferyl-Dglucosidase, ${ }^{1}$ in tissue obtained by muscle biopsy or cultured skin fibroblasts. In the past, measurement of GAA activity in the blood has been problematic. The enzyme is expressed in lymphocytes, but mature neutrophils contain another enzyme, maltase glucoamylase, which has some overlapping activity with GAA. As it can be very difficult to obtain a pure lymphocyte sample, false negatives can occur. It was recognized that acarbose inhibits maltase glucoamylase and its addition to a whole blood sample results in a reliable measurement of GAA activity. ${ }^{25}$ This has led to the development of a simple blood filter paper sample, which can be mailed directly to the reference laboratory, where the blood is extracted and processed for the enzyme assay. This same method has been adapted for newborn screening.

In the later-onset patients, the diagnostic evaluation has typically been by muscle biopsy; ${ }^{26,27}$ however, there is a significant false negative rate because muscle fibers sporadically accumulate glycogen, so it is possible to sample normal tissue. On microscopy, glycogen is periodic-acid Schiff stain-positive, though glycogen can be washed out during processing, and the empty vacuoles may not be readily recognized. Molecular testing may be used to confirm the diagnosis after an abnormal enzyme assay result or after an abnormal muscle biopsy. Magnetic resonance imaging studies $^{28}$ have shown that there is a consistent sequence of muscle changes in patients with LOPD, and may help distinguish Pompe disease from other limb-girdle muscular dystrophies. In patients with known disease, ${ }^{29}$ nuclear magnetic resonance imaging is able to quantitate the rate of fatty degenerative changes per year.

Genotype information can be used for family counseling and potentially for prenatal diagnosis when the mutations in the family are known, such as from having a previously affected child. The presence of known severe or mild mutations may inform the phenotype and prognosis, for example, patients with at least one copy of the common splice-site mutation generally have a milder disease while those with CRIM-negative mutations have severe disease.

A diagnostic workup depends on the age of presentation.

Where newborn screening is available, it is fairly straightforward. A chest X-ray will show cardiomegaly and follow-up two-dimensional (2D)-echocardiography will show evidence of left ventricular hypertrophy in IOPD. The GAA deficiency can be confirmed on leucocyte assay; a skin biopsy for fibroblast culture and CRIM status determination is advisable.

The evaluation of hypertrophic cardiomyopathy in the first few months of life, especially with associated neck and truncal hypotonia, should have GAA activity measure (leucocytes or filter paper sample). Similarly, infants being evaluated for hypotonia and poor feeding should have a chest $\mathrm{X}$-ray and further evaluation with GAA activity if there is evidence of cardiomegaly.

Older patients with evidence of proximal limb-girdle weakness and/or pulmonary dysfunction or disordered breathing should have GAA activity measured. Muscle magnetic resonance imaging may also be helpful to support the diagnosis and determine the degree of muscle involvement.

\section{Therapy development}

Early attempts at ERT ${ }^{1}$ were not successful. A trial in three infants with Pompe disease with an enzyme derived from the fungus Aspergillus niger showed uptake of the enzyme in the liver, but not clearance from skeletal muscle. The patients also developed an immune response. Similarly, treatment with human placenta-derived GAA did not provide a sustained 
effect. Following the cloning of the GAA cDNA, and the recognition of the importance of enzyme uptake via the mannose-6-phosphate receptors, recombinant human GAA (rhGAA) was able to be produced in Chinese hamster ovary cell lines and from the milk of transgenic animals, including rabbits. The earliest clinical trials ${ }^{1}$ were carried out in infants over 36 weeks, using intravenous infusion of rabbit milkderived GAA. Results showed an increase in skeletal muscle enzyme activity and improved muscle function. Ultimately, a Chinese hamster ovary-derived enzyme, which was able to be produced more efficiently, was used for further clinical development, regulatory approval studies, and subsequent commercial supply.

Myozyme (alglucosidase alpha) was approved in 2006 in the USA and EU, and is now commercially available in many industrially developed countries. Later, in the USA, Lumizyme (GAA), made in a scaled up manufacturing process, was not considered by the US Food and Drug Administration to be equivalent to Myozyme. It was initially approved for use in patients over 8 years of age, and in 2014 was approved for all ages.

The clinical development of rhGAA was unique in that the first clinical trials were in infants because of the great unmet medical need and high mortality rate in untreated infants. Typically, new drugs are initially developed and gain regulatory approval for adult patients before studies are done in children.

\section{Infant-onset Pompe disease}

Two open-label studies were carried out to determine the efficacy of rhGAA (Myozyme). One study was of 18 infants,,$^{30}$ aged 6 months or less at the time enrollment, treated with bi-weekly infusions for 52 weeks, at either 20 or $40 \mathrm{mg} / \mathrm{kg} /$ day. The primary clinical endpoint was survival, compared to a historical control group. At 18 months of age, all of the infants were alive, a reduction in risk of death by $99 \%$. By the end of the study, however, six infants required ventilator support, representing a reduction by $88 \%$. The second study ${ }^{31}$ was in 21 infants, aged between 3 and 43 months at the time of enrollment; therefore, some of these infants had more advanced disease at enrollment. At study end, 71\% (15/21) were alive and $44 \%(7 / 16)$ were free of invasive ventilation, showing a reduced risk of death by $79 \%$ and ventilator-dependence by $58 \%$. No benefit was demonstrated for a dose of $40 \mathrm{mg} / \mathrm{kg}$ dose over the $20 \mathrm{mg} / \mathrm{kg}$ dose, the ultimately approved dose.

\section{Late-onset Pompe disease}

A Phase III pivotal randomized double-blind placebo-controlled study in LOPD ${ }^{32}$ was completed in 2007 in 90 patients aged
8 years and older and who were ambulatory and free of invasive ventilation; 60 patients received the drug and 30 placebo. After 78 weeks of treatment $(20 \mathrm{mg} / \mathrm{kg})$, subjects showed improvement in the primary endpoints, 6-minute walk test and forced vital capacity. Hypersensitivity reactions occurred in 5\%-8\%. Antibody sero-conversion occurred in all patients with available complete data (59/60); median peak titers were at 12 weeks of treatment and tended to decrease thereafter; no patients tested positive for inhibition of enzyme activity, but 18 tested positive for inhibition of enzyme uptake.

\section{Treatment}

Currently, there is one approved drug for the treatment of Pompe disease: ERT with intravenous infusion of rhGAA (Myozyme, Lumizyme). The development of this drug, noted earlier, followed the precedent of other ERTs for lysosomal storage diseases (eg, recombinant glucocerebrosidase for Gaucher disease).

In some countries, ERT may be available for treatment of IOPD, but not for LOPD, possibly because of concern about the cost of treating a slowly progressive disease.

\section{Outcomes}

Treatment outcomes depend largely on CRIM status, age at initiation of treatment, severity of the muscle involvement (fibrotic muscle cannot regenerate), muscle fiber distribution, and degree of autophagy in muscle cells, as discussed in the "Pathology" section.

A subset of the most severely affected infants (CRIM-negative) have less successful response to ERT. ${ }^{33}$ In a retrospective review of eleven CRIM-negative and 21 CRIM-positive infants, Kishnani et $\mathrm{al}^{33}$ showed that by 27 months of age, all of the CRIM-negative infants had died or became ventilator dependent but only 4/21 (19\%) of the CRIM-positive infants had a similar outcome. Anti-GAA immunoglobulin (IgG) antibody levels were increased earlier and reached a higher level in the CRIM-negative infants; analysis of neutralizing antibody by assessing inhibition of enzyme activity was positive in $2 / 5$ and inhibition of enzyme uptake was positive in $3 / 5$ of these infants, suggesting that outcome is related to the immune-mediated response to the exogenous protein. Immune modulation therapy ${ }^{34}$ with retuximab and methotrexate has resulted in rapid clearance of antibody in a CRIM-negative infant and some clinical improvements with continued ERT. Two additional patients with elevated antibody titers after initiation of ERT and two patients ${ }^{35}$ treated concomitantly with the initiation of ERT were reported to be immune-tolerant and making clinical progress. 
In patients with established highly elevated antibody titers, response to the standard immune modulation regimen may not be successful. ${ }^{36}$ Some CRIM-positive infants and late-onset patients ${ }^{37}$ can develop high-sustained antibody levels that may impact ERT efficacy. The addition of bortezomib, ${ }^{38,39}$ a protease inhibitor that depletes plasma cells, has resulted in reduction of high-sustained antibody titers in both CRIM-negative and CRIM-positive patients.

In a recent report of 33 IOPD patients in the UK, ${ }^{40}$ 13/29 (45\%) were CRIM-negative, of whom nine underwent immunomodulation therapy (one CRIM-positive patient was treated because of a sibling history of severe infusion-associated reactions) prior to initiation of ERT. The mean follow-up time was 4 years, 1.5 months (range: 6 months-13.5 years). At baseline, $79 \%$ had heart failure, $66 \%$ were failing to thrive, and $70 \%$ had evidence of focal pulmonary collapse. At follow-up, $60 \%$ were ventilatorfree and 30\% were ambulatory. The higher than previously reported number of CRIM-negative patients in this cohort may explain the heterogeneity of outcomes. Details of patients identified by newborn screening and treated presymptomatically are discussed below.

In LOPD, a retrospective and prospective study of 62 patients ( 59 treated) $)^{41}$ in the UK, followed for up to 3 years, there was a significant improvement in the 6-minute walk test and muscle strength; there was no correlation between time on ERT and respiratory function or body mass index.

Van der Meijden et $\mathrm{al}^{42}$ reported the largest cohort of patient-reported outcomes, from the International Pompe Association, from 408 patients (seven primary participating countries) during 2002-2013. They describe the natural history of the disease with impact on daily functioning and quality of life. In 268 untreated patients, the 5-year survival rate from diagnosis was $98 \%$ and the respective 10-, 20-, and 30 -year survival rates were $83 \%, 65 \%$, and $40 \%$. In a survival model in 283 patients on ERT, it was estimated that at any given time, a patient on ERT had a 59\% less risk of dying than an untreated patient.

\section{Other therapies}

\section{Diet therapy}

Supportive treatment with a low carbohydrate, high protein diet, and aerobic exercise ${ }^{43}$ may help prevent decline by decreasing glycogen storage and improving fatty acid oxidation but will not impact irreversible muscle damage. Treatment with a high protein diet and 1-alanine supplementation in late-onset patients ${ }^{44}$ did not have a significant effect.

\section{Gene therapy}

Early attempts at gene therapy with adenovirus and adenoassociated virus (AAV) vectors in the Pompe disease mouse model were mostly unsuccessful due in part to nonsustained expression of enzyme over time and/or immune responses. Further modification of the AAV vectors with specific serotypes that have less immunogenicity and the addition of tissue specific promoters, such as for the liver, ${ }^{45}$ have resulted in a high degree of efficacy in mouse models. There are currently many ongoing clinical trials ${ }^{46}$ utilizing AAV gene delivery systems.

A Phase I/II clinical trial of intra-diaphragmatic delivery of recombinant AAV1-human GAA (rAAV1-human GAA) ${ }^{47}$ was carried out in five ventilator-dependent children. Following injection, all subjects showed improvements in respiratory function, including increased length of time tolerated off assisted ventilation.

In humans, however, prior exposure to the AAV in early life ${ }^{45}$ can result in a potent humoral immune response when treated with an AAV therapeutic, inducing neutralizing antibodies that can impact efficacy and may compromise repeat dosing if necessary. In order to mitigate this risk, Corti et a ${ }^{48}$ have developed a strategy to utilize an immune modulation protocol with pre- or coadministration of rituximab and sirolimus (previously shown to ablate B-cells in Pompe patient who had a high antibody response to ERT) with adeno-associated virus-mediated gene transfer of wild-type desmin cDNA (AAV-DES)-human GAA in a GAA mouse model. This strategy is being evaluated on single and repeat vector dosing preclinical studies (rodents and nonhuman primates) to assess the production of neutralizing antibodies, bio-distribution of the vector, and whether this strategy might enable readministration of the same AAV vector over time. Presumably, if shown to be safe, they will proceed with a proposed Phase I/II study in patients with LOPD, injecting the tibialis anterior muscle.

Other viral vectors have also been explored. Recently, ${ }^{49}$ lentiviral-mediated therapy has shown some promise in neonatal murine models of mucopolysaccharidosis type I. A similar approach using the lentiviral vector encoding human GAA in a neonatal murine Pompe disease model $^{50}$ has resulted in sustained expression after 24 weeks with clearance of glycogen and no significant immune response.

Another approach ${ }^{51}$ has been to genetically modify hematopoietic stem cells ex vivo using a lentiviral-mediated vector to overexpress human GAA. The cells were then transplanted back to the donor mice with $\sim 35 \%$ engraftment, glycogen clearance, and increased exercise tolerance. 
Douillard-Guilloux et $\mathrm{al}^{52}$ used lentiviral vector-mediated short hairpin RNA to target the muscle form of the glycogen synthase enzyme and reduce endogenous production of glycogen. The mice showed decreased accumulation of glycogen and improved exercise tolerance.

\section{Enhanced ERT}

While the currently approved rhGAA can significantly improve cardiac disease, there may still be progression of weakness, particularly in the skeletal muscle, which primarily utilizes the cation-independent mannose-6-phosphate receptor for enzyme uptake. ${ }^{53}$ In order to take advantage of this fact and provide improved delivery of rhGAA to the lysosome, new technologies have been explored.

Neo-rhGAA has been chemically engineered ${ }^{54}$ to increase enzyme uptake by the addition of mannose-6-phosphate residue-bearing synthetic oligosaccharides, resulting in increased cellular uptake and glycogen clearance in the Pompe disease mouse model. A clinical trial ${ }^{55}$ is currently underway to assess the safety and efficacy in repeat dosing of neoGAA in patients with LOPD.

A fusion protein ${ }^{56}$ has been developed that utilizes glycosylation-independent lysosomal targeting. The protein contains rhGAA fused with a portion of insulin-like growth factor II, which has a higher affinity for the cation-independent mannose-6-phosphate receptor. In the Pompe mouse model, there has been a significant increase in glycogen clearance in skeletal muscle.

A Phase I/II study in 21 patients with LOPD $^{57}$ treated for 24 weeks showed improvement in pulmonary function (maximum inspiratory and expiratory pressures) and endurance (6-minute walk test). These improvements were maintained in an extension study. Hypoglycemia was noted (due to increased insulin-like growth factor II activity), but was transient, and managed with increased calorie intake. Two patients withdrew from the study due to infusion-related reactions. A Phase III study of safety and efficacy is underway.

\section{Chaperone therapy}

Small molecule pharmacologic chaperones are currently ${ }^{58-60}$ being evaluated as therapeutic options in several single gene disorders, including lysosomal storage disorders, where the pathology can be attributed to a misfolded but still functional enzyme, albeit, with reduced activity. The misfolded mutant enzyme is recognized as aberrant by the quality control system in the endoplasmic reticulum of the cell, and is subsequently tagged for degradation, or, in some cases, retention in the endoplasmic reticulum.
The chaperones thus far studied in Pompe disease ${ }^{61}$ are the imino sugars, deoxynojirimycin and N-butyldeoxynojirimycin, which bind to the active site of the enzyme. The chaperone acts by stabilizing the enzyme after translation so that it is able to exit the endoplasmic reticulum, rather than be tagged for degradation and can then be targeted normally to the end organelle, in the case of Pompe disease, the lysosome. There, the chaperone dissociates, allowing the enzyme to clear substrate. The mutations that result in a misfolded enzyme are typically missense or small insertions or deletions.

In preclinical studies in the Pompe disease murine model and in human fibroblasts, the chaperone, deoxynijirimycin (DNJ), was able to enhance the activity of the mutant enzyme. Subsequently, a phase II study of DNJ (Amicus Therapeutics AT2220, NCT00688597) was initiated in adult patients with Pompe disease. ${ }^{62,63}$ Unfortunately, the first three patients enrolled showed an increase in muscle weakness and biomarkers, consistent with disease progression; the study was prematurely terminated. In order to understand the results of this study, a study of the muscle pharmacokinetics of AT2220 was carried out in normal healthy volunteers and showed that the intramuscular half-life of the chaperone was greater than anticipated compared to plasma and thus would effectively cause prolonged inhibition of GAA. It was also observed that the pharmacologic chaperone could stabilize the recombinant enzyme, and when used as an adjunct to ERT, could potentially increase the plasma half-life and allow for greater tissue penetration.

\section{Newborn screening}

Newborn screening for inborn errors of metabolism has been one of the great public health successes since its introduction in the 1960s in most of the industrialized world. In the last 10 years, there has been a significant increase in the number of disorders that can be screened because of advancements in the technology used for high-throughput screening of newborn blood filter paper samples. In 2004, the American College of Medical Genetics ${ }^{64}$ surveyed physicians with expertise in these disorders and other interested parties to determine a consensus for a uniform panel. Two of the major criteria for inclusion were availability of a suitable test and treatment. At that time, ERTs were available or in development, but a suitable test was not. Currently, methods are available and are being evaluated in pilot studies ${ }^{65}$ : a fluorometric enzyme assay (a miniaturization of the method discussed earlier); a tandem mass spectrometry method for measuring residual enzyme activity, multiplexed with assays for four other lysosomal storage disorders ${ }^{66}$; a protein quantification method has also been developed, ${ }^{67}$ but not 
introduced; and a digital microfluidics enzyme assay ${ }^{68}$ method where discrete micro droplets of blood are manipulated under an electric field on a microchip.

The data from a pilot study, utilizing the fluorometric assay in Taiwan, ${ }^{16}$ are impressive. Chien et al ${ }^{69}$ were able to show that four infants with IOPD (evidence of cardiomegaly, left ventricular hypertrophy, and generalized accumulation of quadriceps muscle glycogen) detected and treated early (before the age of 1 month) showed reversal of cardiac disease and normal growth and development at the time of reporting. A more recent report ${ }^{70}$ of these four patients and an additional six IOPD children, followed for 28-90 months, confirmed the rapid response of the cardiomyopathy by 6 months of treatment, remaining stable. All were ventilator-free. All, however, showed slower motor development after approximately 2 years of age, with proximal muscle weakness and orofacial symptoms (facial weakness, nasal speech, hearing loss); three patients had mild cognitive delays (requiring special help with language and mathematics). All patients developed low titers of anti-rhGAA antibody that declined over time. All of these patients were CRIM-positive.

Thirteen later-onset Pompe disease patients ${ }^{71}$ were also detected by newborn screening (no evidence of cardiac involvement) and followed for up to 4 years. Four patients were started on ERT between the ages of 1.5 and 3 years because of onset of hypotonia and delayed motor milestones (no outcomes were reported). The other nine patients continue to be followed every 3-6 months.

In the USA, Pompe disease was recently evaluated and recommended, in March 2015, to be added to the universal screening panel by the federal Secretary's Advisory Committee on Heritable Disease in Newborns and Children, in the Department of Health and Human Services. The final decision, however, is made by individual states. Pompe disease is currently screened or in the process of being implemented, in several states. Several other states are considering adding it to their current newborn screening panels, but have not yet been approved. Two different methods are being used, tandem mass spectrometry assays multiplexed with assays for other lysosomal storage disorders and digital microfluidics.

Results were recently published from the state of Missouri, USA, ${ }^{72}$ for the first 6 months of screening by digital microfluidics for Pompe, Gaucher, and Fabry diseases, and mucopolysaccharidosis type I. A total of 43,701 samples were screened, with 18 positive for Pompe disease; six were subsequently confirmed to have Pompe disease, three infant-onset and three late-onset disease; two were found to carry a pseudo-deficiency mutation, three were carriers, two had genotypes of unknown significance, and four were false positives. The incidence rate of 1:5,463 is much higher than the generally accepted published incidence of 1:40,000, but similar to the reported incidence in a Hungarian study ${ }^{73}$ of 40,024 samples in which nine cases were confirmed, 25 were carriers, 27 were normal (false positives), and three had a genotype of unclear significance.

\section{Summary}

Pompe disease is a rare autosomal recessive genetic disorder due to a deficiency of the lysosomal hydrolase enzyme, GAA, caused by mutations in the $G A A$ gene. It presents with a broad phenotypic spectrum. The advent of ERT with recombinant GAA has resulted in significant improvements in morbidity and mortality, although some patients continue to decline despite therapeutic intervention. New strategies are being developed to improve delivery of enzyme through various methods to alter the natural history of the disease. Newborn screening has shown that early therapeutic intervention, before irreversible muscle damage has occurred, may provide the greatest clinical benefit.

\section{Disclosure}

The authors report no conflicts of interest in this work.

\section{References}

1. Hirschhorn R, Reuser AJJ. Glycogen storage disease type II: acid $\alpha$-glucosidase (acid maltase) deficiency. In: Scriver CR, Beau$\operatorname{det}$ AL, Valle D, Sly WS, editors. The Metabolic and Molecular Bases of Inherited Disease. 8th ed. New York: McGraw Hill; 2001: 3389-3420.

2. Kroos M, Hoogeveen-Westerveld M, Michelakakis H, et al. Update of the Pompe disease mutation database with 60 novel sequence variants and additional studies on functional effect of 34 previous reported variants. Hum Mut. 2012;33(8):1161-1165.

3. Moreland RJ, Xioying J, Jin X, Zhang K, et al. Lysosomal acid $\alpha$-glucosidase consists of four different peptides processed from a single chain precursor. J Biol Chem. 2005;280(8):6780-6791.

4. Kroos MA, Mullaart RA, Van Vliet L, Pomponio RJ, Amartino H, Kolodny EH. p.[C576S: E689K]: pathogenic combination or polymorphism in Pompe disease? Eur J Hum Genet. 2008;16(8):875-879.

5. Huie ML, Hirschhorn R, Chen AS, Martiniuk F, Zhong N. Mutation at the catalytic site (M519V) in glycogen storage disease type II (Pompe disease). Hum Mutat. 1994;4(4):291-293.

6. Boerkoel CF, Exelbert R, Nicastri C, et al. Leaky splicing mutation in the acid maltase gene is associated with delayed onset of glycogenosis type II. Am J Hum Genet. 1995;56:887-897.

7. Pompe Center, Erasmus Medical Center, Rotterdam, NL. Available from: www.pompecenter.nl. Accessed on April 20, 2016.

8. Askanas V, Engel WK, DiMauro S, Brooks BR, Mehler M. Adult onset acid maltase deficiency-morphologic and biochemical abnormalities reproduced in cultured muscle. N Engl J Med 1976;294:573-578.

9. Griffen JL. Infantile acid maltase deficiency. I. Muscle fiber destruction after lysosomal rupture. Virchows Arch B Cell Pathol Incl Mol Pathol. 1984;45(1):23-36.

10. Shea L, Raben N. Autophagy in skeletal muscle: implications for Pompe disease. Int J Clin Pharmacol Ther. 2009;47 (Suppl 1):S42-S47. 
11. Raben N, Baum R, Schreiner C, et al. When more is less. Excess and deficiency of autophagy coexist in skeletal muscle in Pompe disease. Autophagy. 2009;5(1):111-113.

12. Lim JA, Li L, Kakhlon O, Myerowitz R, Raben N. Defects in calcium homeostasis and mitochondria can be reversed in Pompe disease. Autophagy. 2015;11(2):385-402.

13. Falk DJ, Todd AG, Lee S, et al. Peripheral nerve and neuromuscular junction pathology in Pompe disease. Hum Mol Genet. 2015;24(3): 625-636.

14. Hobson-Webb LD, Austin SL, Jain S, Case LE, Greene K, Kishnani PS. Small-fiber neuropathy in Pompe disease: first reported cases and prospective screening of a clinic cohort. Am J Case Rep. 2015;16: 196-201.

15. Byrne BJ, Kishnani PS, Case LE, et al. Pompe disease: design, method and early findings from the Pompe Registry. Mol Genet Metab. 2011;103(1):1-11.

16. Chien YH, Lee NC, Huang HJ, Thurberg BL, Tsai FJ, Hwu WL. Lateonset Pompe disease: early detection and early treatment initiation enabled by newborn screening. J Pediatr. 2011;158:1023-1027.

17. Shieh JJ, Wang LY, Lin CY. Point mutation in Pompe disease in Chinese. $J$ Inherit Metab Dis. 1994;17(1):145-148.

18. Kumamoto S, Katatuchi S, Nakamura K, et al. High frequency of acid alpha glucosidase pseudodeficiency complicates newborn screening for glycogen storage disease in the Japanese population. Mol Genet Metab. 2009;97(3):190-195.

19. Labrousse P, Chien YH, Pomponio RJ, et al. Genetic heterozygosity and pseudodeficiency in the Pompe disease newborn screening pilot program. Mol Genet Metab. 2010;99(4):379-383.

20. Hagemans M, Winkel L, Van Doorn P, et al. Clinical manifestations and natural course of late-onset Pompe disease in 54 Dutch patients. Brain. 2005;128:671-677.

21. Amartino H, Panceira D, Pomponio RJ, et al. Two clinical forms of glycogen-storage disease type II in two generations of the same family. Clin Genet. 2006;69(2):187-188.

22. Kroos MA, Pomponio RJ, Hagermans ML. Broad spectrum of Pompe disease in patients with the same c.-32-13 T $>$ G haplotype. Neurology. 2007;68(2):110-115

23. Van den Hout HM, Hop W, van Diggelen OP, et al. The natural course of Pompe's disease: 20 original cases compared to 133 cases from the literature. Pediatrics. 2003;112(2):332-340.

24. Bali DS, Tolun AA, Goldstein JL, Dai J, Kishnani PS. Molecular analysis and molecular processing in late-onset Pompe disease patients with low levels of acid $\alpha$-glucosidase activity. Muscle Nerve. 2011;43(5):665-670.

25. Winchester B, Bali DS, Bodamer OA, et al; Pompe Disease Diagnostic Working Group. Methods for a prompt and reliable laboratory diagnosis of Pompe disease: report from an international consensus meeting. Mol Genet Metab. 2008;93(3):275-281.

26. American Association of Neuromuscular and Electrodiagnostic Medicine. Diagnostic criteria for late-onset (childhood and adult) Pompe disease. Muscle Nerve. 2009;40:149-160.

27. Vissing J, Lukacs Z, Straub V. Diagnosis of Pompe disease: muscle biopsy vs blood-based assays. JAMA Neurol. 2013;70(7):923-927.

28. Pichiecchio A, Ugetti C, Ravaglia S, et al. Muscle MRI in adult-onset acid maltase deficiency. Neuromuscl Disord. 2004;14:51-55.

29. Carlier PG, Azzabou N, deSousa PL, et al. Skeletal muscle quantitative nuclear magnetic resonance imaging follow up of adult Pompe patients. J Inherit Metab Dis. 2015;38(3):565-572.

30. Kishnani PS, Corzo D, Nicolino M, et al. Recombinant human acid [alpha]-glucosidase: major benefits in infantile-onset Pompe disease. Neurology. 2007;68(2):99-109.

31. Nicolino M, Byrne B, Wraith JE, et al. Clinical outcomes after long-term treatment with alglucosidase alfa in infants and children with advanced Pompe disease. Genet Med. 2009;11(3):210-219.

32. Van der Ploeg AT, Clemens PR, Corzo D, et al. A randomized study of alglucosidase alpha in late-onset Pompe's disease. $N$ Engl J Med. 2010;362:1396-1406.
33. Kishnani PS, Goldenberg PC, DeArmey SL, et al. Cross-reactive immunologic material status affects treatment outcomes in Pompe disease infants. Mol Genet Metab. 2010;99:26-33.

34. Mendelsohn NJ, MessengerYH, Rosenberg AS, Kishnani PS. Elimination of antibodies to recombinant enzyme in Pompe disease. $N$ Engl J Med. 2009;360(2):194-195.

35. Messinger YH, Mendelsohn NJ, Rhead W, et al. Successful immune tolerance induction to enzyme replacement therapy in CRIM-negative infantile Pompe disease. Genet Med. 2012:14(1);135-142.

36. Banugaria SG, Prater SN, Ng YK, et al. The impact of antibody on clinical outcomes in diseases treated with therapeutic protein: lessons learned from infantile onset Pompe disease. Genet Med. 2011;13(8):729-737.

37. Patel TT, Banugaria SG, Case LE, Wenninger S, Schoser B, Kishnani PS. The impact of antibody in late-onset Pompe disease: a case series and literature review. Mol Genet Metab. 2012;106(3):301-309.

38. Banugaria SG, Prater SN, McGann JD, et al. Bortezomib in the rapid reduction of high antibody titers in disorders treated with therapeutic protein: lessons learned from Pompe disease. Genet Med. 2013; 15(2):23-31.

39. Stenger EO, Kazi Z, Lisi E, Gambello M, Kishnani PS. Immune tolerance strategies in siblings with infantile Pompe disease - advantages for a preemptive approach to high-sustained antibody titers. Molec Genet Metab Rep. 2012;4:30-34

40. Broomfield A, Fletcher J, Davison J, et al. Response of 33 UK patients with infantile-onset Pompe disease. J Inherit Metab Dis. 2016;39(2):261-271.

41. Anderson LJ, Henley W, Wyatt KM, et al. Effectiveness of enzyme replacement therapy in patients with late-onset Pompe disease: results from the NCS-LSD cohort study. J Inherit Metab Dis. 2014;37(6):945-952.

42. Van der Meijden JC, Gungor D, Kruijshaar ME, Muir AD, Broekgaarden HA, Van der Ploeg AT. Ten years of the International Pompe survey: patient reported outcomes as a reliable tool for studying treated and untreated children and adults with non-classic Pompe disease. J Inherit Metab Dis. 2015;38(3):495-503.

43. Slonim AE, Coleman RA, McElligot MA, et al. Improvement of muscle function in acid maltase deficiency by high-protein therapy. Neurology. 1983;33(1):34-38.

44. Bodamer OA, Halliday D, Leonard JV. Effect of 1-alanine supplements in late-onset glycogen storage disease type II. Neurology. 2000;55(5):710-712.

45. Calcedo R, Wilson JM. Humoral immune response in AAV. Front Immunol. 2013;4:341.

46. ClinicalTrials.gov. US National Institute of Health. www.clinicaltrials. gov.

47. Smith BK, Collins SW, Conlon TJ, et al. A phase I/II trial of adenoassociated virus mediated alpha-glucosidase gene therapy to the diaphragm for chronic respiratory failure in Pompe disease: initial safety and ventilation outcomes. Hum Gene Ther. 2013;24(6):630-640.

48. Corti M, Elder ME, Falk, DJ, et al. B-cell depletion is protective against anti-AAV capsid immune response: a human case study. Mol Ther Methods Clin Dev. 2014;1. pii: 14033.

49. Kobayashi H, Carbonaro D, Pepper K, et al. Neonatal gene therapy of MPS I mice by intravenous injection of a lentiviral vector. Mol Ther. 2005;11:776-789.

50. Kyosen SO, Iizuka S, Kobayashi H, et al. Neonatal gene transfer using lentiviral vector for murine Pompe disease: long-term expression and glycogen production. Gene Ther. 2010;17:521-530.

51. Van Til NP, Stok M, Aerts H, et al. Lentiviral gene therapy of murine hematopoietic stem cells ameliorates the Pompe disease phenotype. Blood. 2010;115(26):5329-5337.

52. Douillard-Guilloux G, Raben N, Takikita S. Restoration of muscle functionality by genetic suppression of glycogen synthesis in a murine model of Pompe disease. Hum Mol Genet. 2010;19(4):684-696.

53. Ghosh P, Griffith J, Gueze HJ, Kornfeld S. Mammalian GGA's act together to sort mannose-6-phosphate receptors. J Cell Biol. 2003;163(4):755-766. 
54. Zhu Y, Jiang JL, Gumlaw PK, et al. Glycoengineered acid alphaglucosidase with improved efficiency at correcting the metabolic aberrations and motor function deficits in a mouse model of Pompe disease. Mol Ther. 2009;17(6):954-963.

55. Safety and efficacy evaluation of repeat NeoGAA dosing in late-onset Pompe disease (NCT01898364). Available from: www.clinicaltrials. gov. Accessed September 15, 2015.

56. Maga JA, Zhou J, Kambampati R, et al. Glycosylation-independent lysosomal targeting of acid $\alpha$-glucosidase enhances muscle glycogen clearance in Pompe mice. J Biol Chem. 2013:288(3);1428-1438.

57. Byrne B, Barohn R, Barshop B, Bratkovic D, Desnuelle C, Henderson R. Lysosomal Disease Network WORLD Symposium 2014; February 10-14, 2014; San Diego, CA.

58. Okumiya T, Kroos MA, Vliet LV, Takeuchi H, Van der Ploeg AT, Reuser AJ. Chemical chaperones improve transport and enhance stability of mutant alpha-glucosidases in glycogen storage disease type II. Mol Genet Metab. 2007;90(1):49-57.

59. Parenti G, Zuppaldi A, Pittis MG, et al. Pharmacological enhancement of mutated $\alpha$-glucosidase activity in fibroblasts from patients with Pompe disease. Mol Ther. 2007;15(3):508-514.

60. Tajima Y, Saito S, Ohno K, Tsukimura T, Tsujino S, Sakuraba H. Biochemical and structural study on a S529V mutant acid $\alpha$-glucosidase responsive to pharmacologic chaperones. J Hum Genet. 2011;56(6):440-446.

61. Flanagan JJ, Rossi B, Tang K, et al. The pharmacologic chaperone 1-deoxynojirimycin increases the activity and lysosomal trafficking of multiple mutant forms of acid-alpha glucosidase. Hum Mutat. 2009;30(12):1683-1692.

62. Khanna R, Powe AC, Lun Y, et al. The pharmacological chaperone AT2220 increases the specific activity and lysosomal delivery of mutant acid alpha-glucosidase, and promotes glycogen reduction in a transgenic mouse model of Pompe disease. PLoS One. 2014;9(7): e102092.
63. Adera M, Boudes P, Bragat A. Lysosomal Disease Network, WORLD Symposium; February 18-18, 2011; Las Vegas, NV.

64. Watson MS, Mann MY, Lloyd-Puryear MA, Rinaldo P, Howell RR. Newborn screening: toward a uniform panel and system. Genet Med. 2006;8:1S-252S

65. Marsden D, Levy H. Newborn screening of lysosomal storage disorders. Clin Chem. 2010;56(7):1071-1079.

66. Gelb MH, Turecek F, Scott CR, Chamoles NA. Direct multiples assay of enzymes in dried blood spots by tandem mass spectrometry for newborn screening of lysosomal storage disorders. J Inherit Metab Dis. 2006;29:397-404.

67. Umapatysivam K, Whittle AM, Ranieri E, et al. Determination of acid $\alpha$-glucosidase protein: evaluation as a screening marker for Pompe disease and other lysosomal storage disorders. Clin Chem. 2000;46(9):1318-1325.

68. Millington DS, Sista R, Eckhardt A et al. Digital microfluidics: a future technology in the newborn screening laboratory? Semin Perinatol. 2010;34(2):163-169.

69. Chien YH, Lee NC, Thurberg BL, et al. Pompe disease in infants: improving prognosis by newborn screening and early intervention. Pediatrics. 2009;124(6):e1116-e1125.

70. Chien YH, Lee NC, Chen CA, et al. Long-term prognosis of patients with infantile-onset Pompe disease diagnosed by newborn screening and treated since birth. J Pediatr. 2015;166(4):985-991.

71. Chien YH, Lee NC, Huang HJ, Thurberg BL, Tsai FJ, Hwu WL. Lateronset Pompe disease: early detection and early treatment initiation enabled by newborn screening. J Pediatr. 2011;158(6):1023-1027.

72. Hopkins PV, Campbell C, Klug T, Rogers S, Raeburn-Miller J, Kiesling J. Lysosomal storage disorder screening implementation: findings from the first 6 months of full population pilot testing in Missouri. J Pediatr. 2015;166(7):172-177.

73. Wittmann J, Karg E, Turi S, et al. Newborn screening for lysosomal storage disorders in Hungary. JIMD Rep. 2012;6:117-125.
Orphan Drugs: Research and Reviews

\section{Publish your work in this journal}

Orphan Drugs: Research and Reviews is an international, peer-reviewed, open access journal publishing original research, reports, reviews and commentaries on all areas of the design and development of orphan drugs for the treatment of rare diseases through to clinical applications. Clinical outcomes, patient safety, and programs for the development and

\section{Dovepress}

effective, safe, and sustained use of medicines will be a feature of the journal. The manuscript management system is completely online and includes a very quick and fair peer-review system, which is all easy to use. Visit http://www.dovepress.com/testimonials.php to read real quotes from published authors. 